\title{
Graphitized hollow carbon spheres and yolk-structured carbon spheres fabricated by metal-catalyst-free chemical vapor deposition
}

\author{
Xufan Li ${ }^{a}$, Miaofang Chi ${ }^{c}$, Shannon M. Mahurin ${ }^{d}$, Rui Liu ${ }^{d}$, Yen-Jun Chuang ${ }^{a}$, Sheng Dai ${ }^{d}$, \\ Zhengwei Pan ${ }^{a, b, *}$ \\ ${ }^{a}$ College of Engineering, University of Georgia, Athens, GA 30602, USA \\ ${ }^{b}$ Department of Physics and Astronomy, University of Georgia, Athens, GA 30602, USA \\ ${ }^{c}$ Center for Nanophase Materials Science, Oak Ridge National Laboratory, Oak Ridge, TN \\ 37831, USA \\ ${ }^{d}$ Chemical Sciences Division, Oak Ridge National Laboratory, Oak Ridge, TN 37831, USA
}

\begin{abstract}
Hard-sphere-templating method has been widely used to synthesize hollow carbon spheres (HCSs), in which the spheres were firstly coated with a carbon precursor, followed by carbonization and core removal. The obtained HCSs are generally amorphous or weakly graphitized (with the help of graphitization catalysts). In this work, we report on the fabrication of graphitized HCSs and yolk-shell Au@ HCS nanostructures using a modified templating method, in which smooth, uniform graphene layers were grown on $\mathrm{SiO}_{2}$ spheres or $\mathrm{Au} @ \mathrm{SiO}_{2}$ nanoparticles via metal-catalyst-free chemical vapor deposition (CVD) of methane. Our work not only provides a new method to fabricate high-quality, graphitized HCSs but also demonstrates a reliable approach to grow quality graphene on oxide surfaces using CVD without the presence of metal catalysts.
\end{abstract}

*Corresponding author. E-mail: panz@uga.edu (Zhengwei Pan). 


\section{Introduction}

In recent years, the synthesis of hollow carbon spheres (HCSs) has attracted considerable attention because of the promising potentials of HCSs in energy storage and conversion [1-8] and in catalytic and adsorption-based application $[1,9,10]$, owning to their many unique features, such as spherical morphology, low density, high specific surface areas, large void space fraction, outstanding thermal and chemical stability, etc. Hard-templating-based methods were widely used to synthesize the HCSs [1]. Typically, the templates, mainly silica spheres [2-6,9-16] and polymer spheres $[7,8,17,18]$ having monodisperse size and spherical shape, were firstly coated with a thin layer of a carbon precursor (e.g., glucose [5-7], resorcinol-formaldehyde resin [3,4,913], dopamine [14,15], and petroleum pitch [16]) to form a core-shell structure, followed by carbonization of the shell and etching removal of the core. The as-synthesized HCSs preserved the morphology and size of the templates, forming negative replicas of the templates. Besides the HCSs, the template approaches also enable to synthesize novel yolk-shell structures, in which one or several movable nanoparticles are encapsulated inside a HCS (e.g., Au@HCS), when core-shell nanoparticles (e.g., $\mathrm{Au} @ \mathrm{SiO}_{2}$ ) are used as the templates [14]. Such yolk-shell carbon structure is beneficial for catalytic and electrochemical applications $[5,6,10,14]$.

The carbon shells of the HCSs fabricated by the templating methods are generally amorphous, which have poor electrical conductivity and are not ideal for some applications such as lithium-ion batteries [1]. To obtain graphitized carbon shells, graphitization catalyst, such as Fe-containing species $[8,18]$, was frequently added into the carbon precursor coating layer in the coating process, which promoted the graphitization of carbon precursor in the carbonization process. However, the obtained carbon shells were only weakly graphitized, exhibiting turbostratic or localized graphitic structures. Herein, we report on the fabrication of graphitized, 
monodisperse HCSs and yolk-shell Au@ HCS nanostructures using a new, modified templating method, in which smooth, uniform graphene layers were grown on $\mathrm{SiO}_{2}$ spheres or $\mathrm{Au} @ \mathrm{SiO}_{2}$ nanoparticles via metal-catalyst-free chemical vapor deposition (CVD) of methane. Our work not only provides a new method to fabricate high-quality HCSs but also demonstrates a reliable approach to grow quality graphene on oxide surfaces using methane CVD without the presence of metal catalysts.

\section{Experimental}

\subsection{CVD fabrication of hollow carbon spheres}

The metal-catalyst-free CVD growth of graphitized HCSs was conducted in a tube furnace system using methane $\left(\mathrm{CH}_{4}\right)$ as the carbon source $[19,20]$. Two kinds of monodisperse $\mathrm{SiO}_{2}$ spheres were used as the templates: micro-sized $\mathrm{SiO}_{2}$ spheres with diameters of $\sim 500 \mathrm{~nm}$ and nano-sized $\mathrm{Au} @ \mathrm{SiO}_{2}$ core-shell spheres with diameters of $\sim 50 \mathrm{~nm}$. The micro-sized $\mathrm{SiO}_{2}$ spheres were synthesized using the Stöber method [21]. The $\mathrm{Au} @ \mathrm{SiO}_{2}$ core-shell nanospheres were prepared using the reported sol-gel method [22]. The spheres were spread on a Si wafer (or other substrates such as aligned carbon nanotube sheets) to form a thin layer of 3-D assembly of silica spheres, which was placed in the center of a horizontal tube furnace. The metal-catalystfree CVD growth of graphitized carbon on $\mathrm{SiO}_{2}$ sphere surfaces requires the growth temperature to be at $1100-1200{ }^{\circ} \mathrm{C}$; below $1100^{\circ} \mathrm{C}$ quality graphitized carbon spheres do not grow $[19,20,23]$, and higher temperature $\left(>1200^{\circ} \mathrm{C}\right)$ can result in the sintering of the spheres [20]. Therefore, in this work, the growth temperature was fixed at $1150{ }^{\circ} \mathrm{C}$. The other synthesis parameters were: methane flow rate, $5 \mathrm{sccm}$ (standard cubic centimeters per minute); Ar flow rate, $150 \mathrm{sccm}$; growth chamber pressure, 150 Torr; and growth time, 1 to $10 \mathrm{~min}$. After growth, 
the samples were immersed in a $1 \mathrm{M} \mathrm{HF}$ aqueous solution for $2 \mathrm{~h}$ to dissolve the $\mathrm{SiO}_{2}$ cores, followed by washing in methanol and drying in an oven at $80^{\circ} \mathrm{C}$ for $1 \mathrm{~h}$.

\subsection{Morphology and structural characterization}

The as-synthesized HCSs and Au@HCS yolk-shell nanostructures were examined using scanning electron microscopy (SEM; FEI Inspect F FEG SEM), transmission electron microscopy (TEM; FEI Titan S aberration-corrected TEM/STEM), and Ramana spectroscopy (a Renishaw Raman 1000 system with a $514 \mathrm{~nm}$ argon ion laser; the laser power is about $1 \mathrm{~mW}$ ).

\section{Results and discussion}

Figure 1a shows a SEM image of the micro-sized $\mathrm{SiO}_{2}$ spheres. The spheres have a spherical shape with a uniform diameter of $\sim 500 \mathrm{~nm}$. After CVD reaction, the $\mathrm{SiO}_{2}$ spheres were uniformly coated with a thin carbon layer, while the spherical shape of the spheres was perfectly preserved and no sintering between the spheres was observed (Fig. 1b). After the $\mathrm{SiO}_{2}$ cores were removed by HF etching, HCSs which are the negative replicas of the $\mathrm{SiO}_{2}$ spheres were obtained. Figures 1c, 1d,e, and 1f show the HCSs obtained after $3 \mathrm{~min}, 5 \mathrm{~min}$, and 8 min of CVD growth, respectively. At shorter growth time ( $<3 \mathrm{~min})$, there have small pores on some spheres, as indicated by the white line arrows in Fig. 1c. Occasionally, carbon hemispheres were observed, as indicated by the hollow arrow in Fig. 1c. However, no collapsed HCSs were observed, showing the rigidity of the spherically shaped spheres. At longer growth time ( $>5$ min), carbon deposition occurred uniformly on the entire surface of each $\mathrm{SiO}_{2}$ sphere, producing HCSs with microscopically smooth outer surfaces (Fig. 1d-f). Figure 1d-f clearly shows that due to the thin, conductive nature of the carbon shells, the HCSs are "transparent" to electron beam and the spheres located at deeper position can be clearly seen in SEM imaging. Moreover, Figure $1 \mathrm{~d}-\mathrm{f}$ also shows that the metal-catalyst-free CVD growth not only precisely replicate 

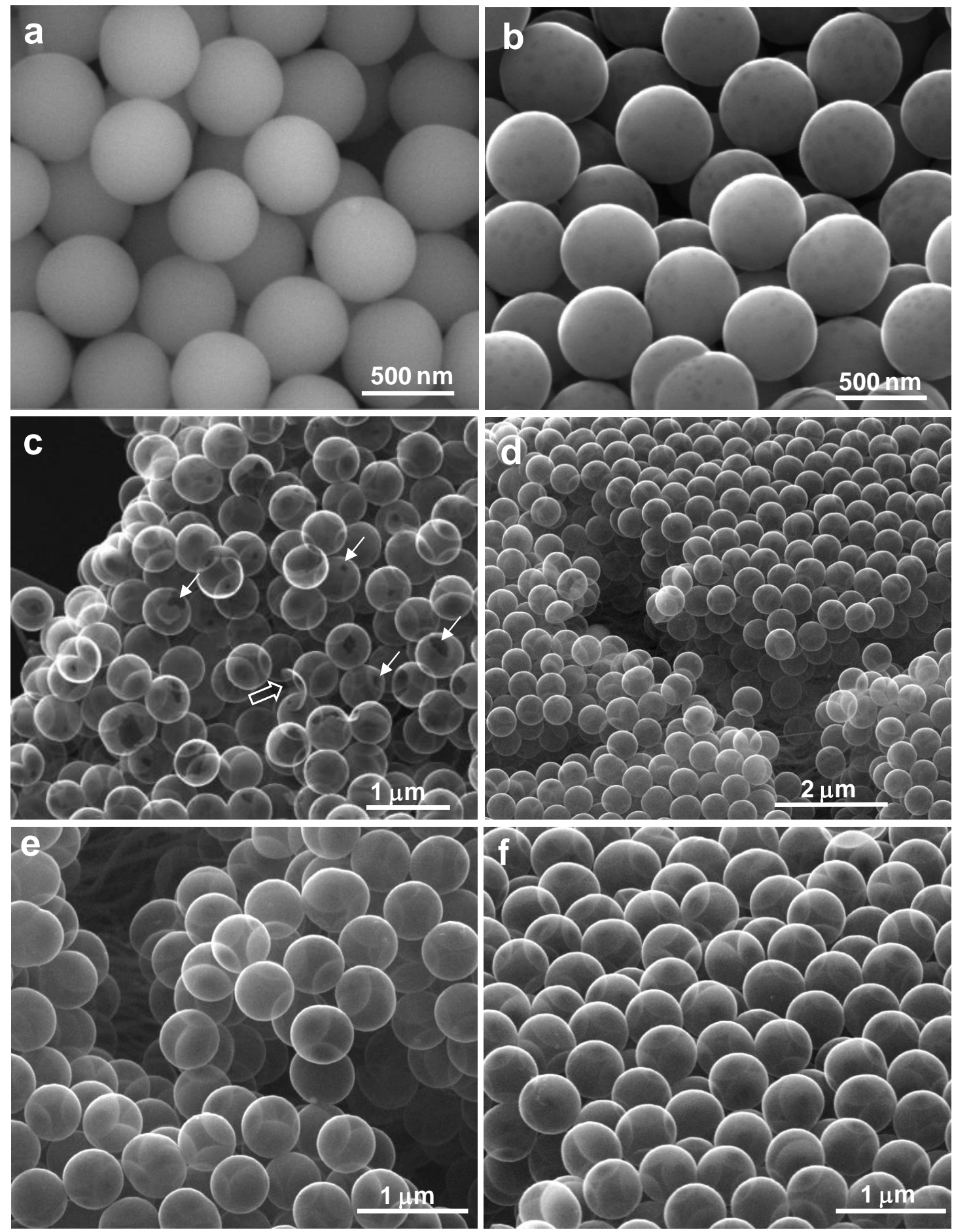

Figure 1. SEM images of HCSs. (a) Micro-sized $\mathrm{SiO}_{2}$ spheres. (b) Carbon-coated $\mathrm{SiO}_{2}$ spheres (after CVD growth). (c) HCSs after 3 min growth. (d,e) HCSs after 5 min growth. (f) HCSs after 8 min growth. The white arrow heads in (c) indicate the holes on spheres. The hollow arrow head in (c) indicates a graphene hemisphere. 
individual $\mathrm{SiO}_{2}$ spheres but also perfectly preserve the 3-D assembly of $\mathrm{SiO}_{2}$ spheres, showing the high penetration and uniform deposition of gaseous hydrocarbons in the CVD process.

The transparency and the spherical shape of the HCSs were verified by TEM/STEM imaging. Figure 2a shows a Z-contrast dark-field STEM image of a cluster of HCSs. In STEM, the hollow spheres resemble bubbles in water, exhibiting a perfectly round cross-section with a uniform shell thickness.
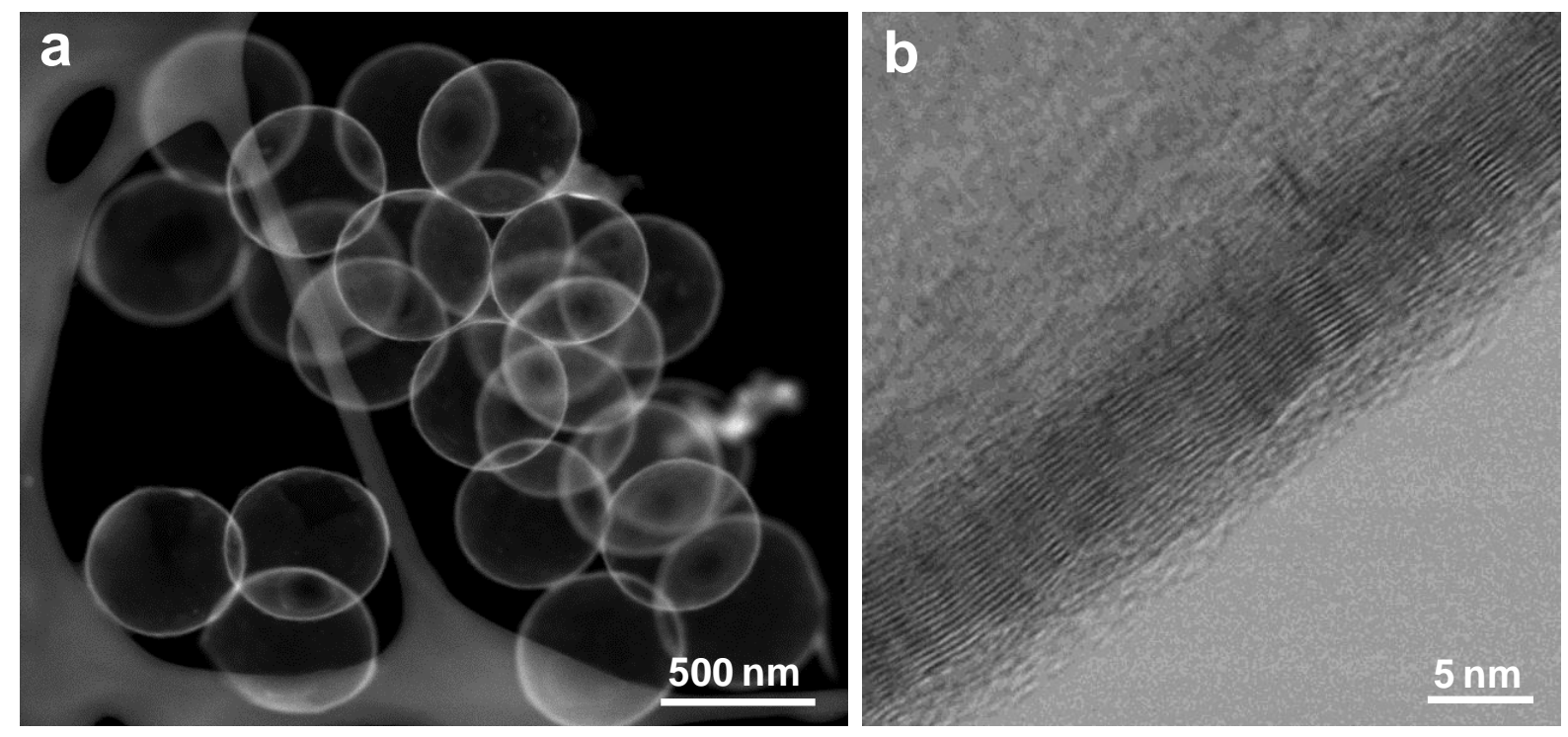

Figure 2. TEM images of HCSs. (a) Z-contrast dark-field STEM image of a cluster of HCSs. (b) HRTEM image of the wall of a HCS.

The microstructures and quality of the shells of the HCSs were examined using highresolution transmission electron microscopy (HRTEM) and Raman spectroscopy. HRTEM imaging (Fig. 2b) shows that the shell is well-graphitized even though no metal catalysts were used, with the graphitization degree comparable to that of the metal-catalyzed CVD multiwall carbon nanotubes [24]. The shell typically consists of $>10$ graphene layers after 5 min of growth. The HRTEM results were further verified by Raman measurements, as the typical Raman 
spectrum shown in Fig. 3a. The Ramana spectrum contains D $\left(\sim 1358 \mathrm{~cm}^{-1}\right), \mathrm{G}\left(\sim 1584 \mathrm{~cm}^{-1}\right), 2 \mathrm{D}$ or G' $\left(\sim 2708 \mathrm{~cm}^{-1}\right)$, and $\mathrm{D}+\mathrm{G}\left(\sim 2928 \mathrm{~cm}^{-1}\right)$ bands. The well-resolved $\mathrm{G}$ band as well as the strong 2D (G') band indicates the existence of significant $s p^{2}$ carbon in the HCSs. The broad 2D band and the low $I_{2 \mathrm{D}} / I_{\mathrm{G}}$ intensity ratio ( 0.7) show the formation of multilayer graphene. The high D band intensity, however, suggests a relatively high level of disorders in the HCSs.
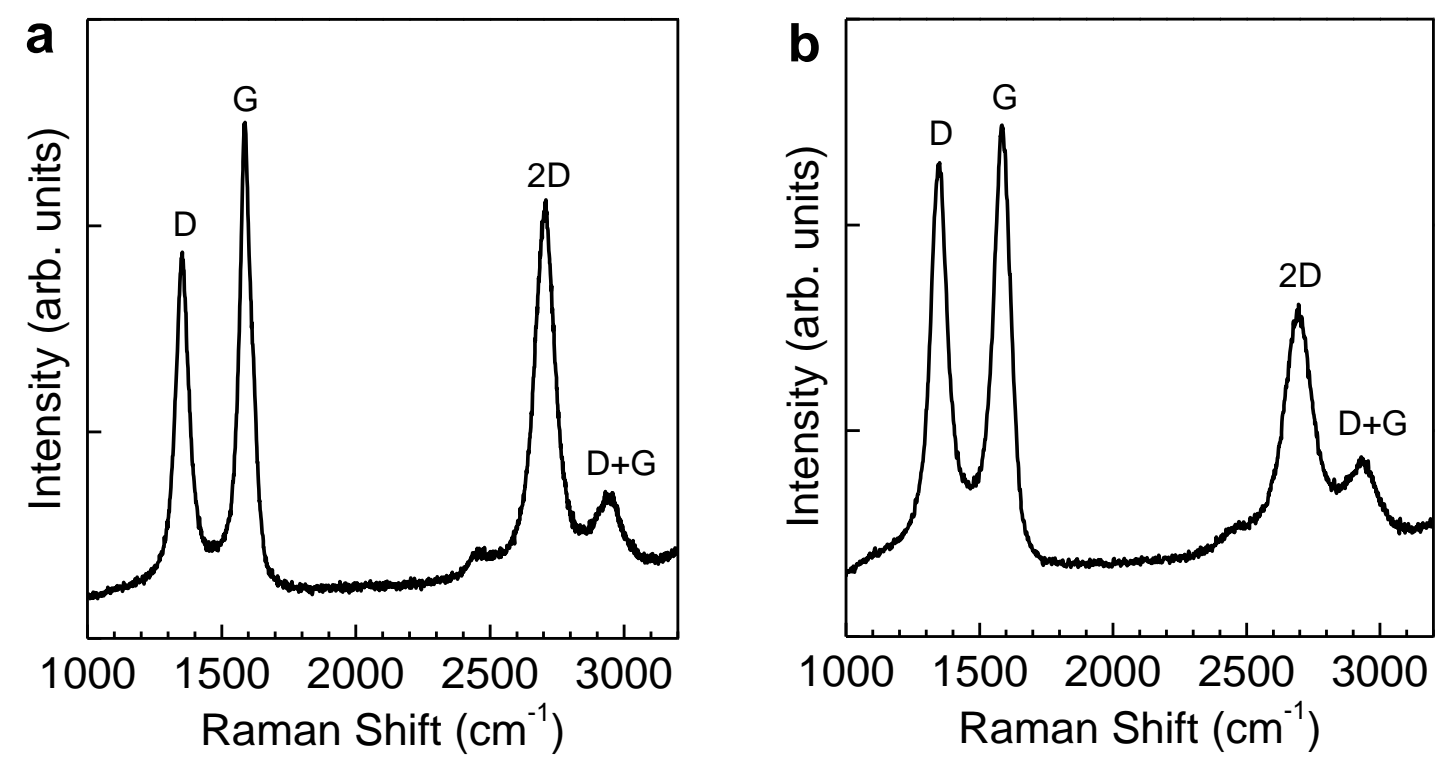

Figure 3. Raman spectra. (a) HCSs. (b) Au@HCS yolk-shell nanostructures.

Besides the microscale HCSs described above, we have also fabricated nanoscale HCSs encapsulating one or several Au nanoparticles, i.e., the Au@ HCS yolk-shell nanostructures, by the metal-catalyst-free $\mathrm{CVD}$ using $\mathrm{Au} @ \mathrm{SiO}_{2}$ core-shell nanoparticles as the templates. Figure 4a shows a SEM image of the $\mathrm{Au} @ \mathrm{SiO}_{2}$ core-shell nanoparticles used in this study. The nanoparticles have a diameter of $\sim 50 \mathrm{~nm}$, and each particle contains one to three $\sim 10 \mathrm{~nm} \mathrm{Au}$ nanoparticles. After CVD reaction followed by HF etching, HCSs that precisely preserved the shapes of the original $\mathrm{SiO}_{2}$ nanoparticles were obtained and the $\mathrm{Au}$ nanoparticles were 
encapsulated inside the spheres, as shown in Fig. 4b. Notably, despite the small size of the $\mathrm{Au} @ \mathrm{SiO}_{2}$ nanoparticles, there is no apparent agglomeration of the particles during the hightemperature CVD growth.
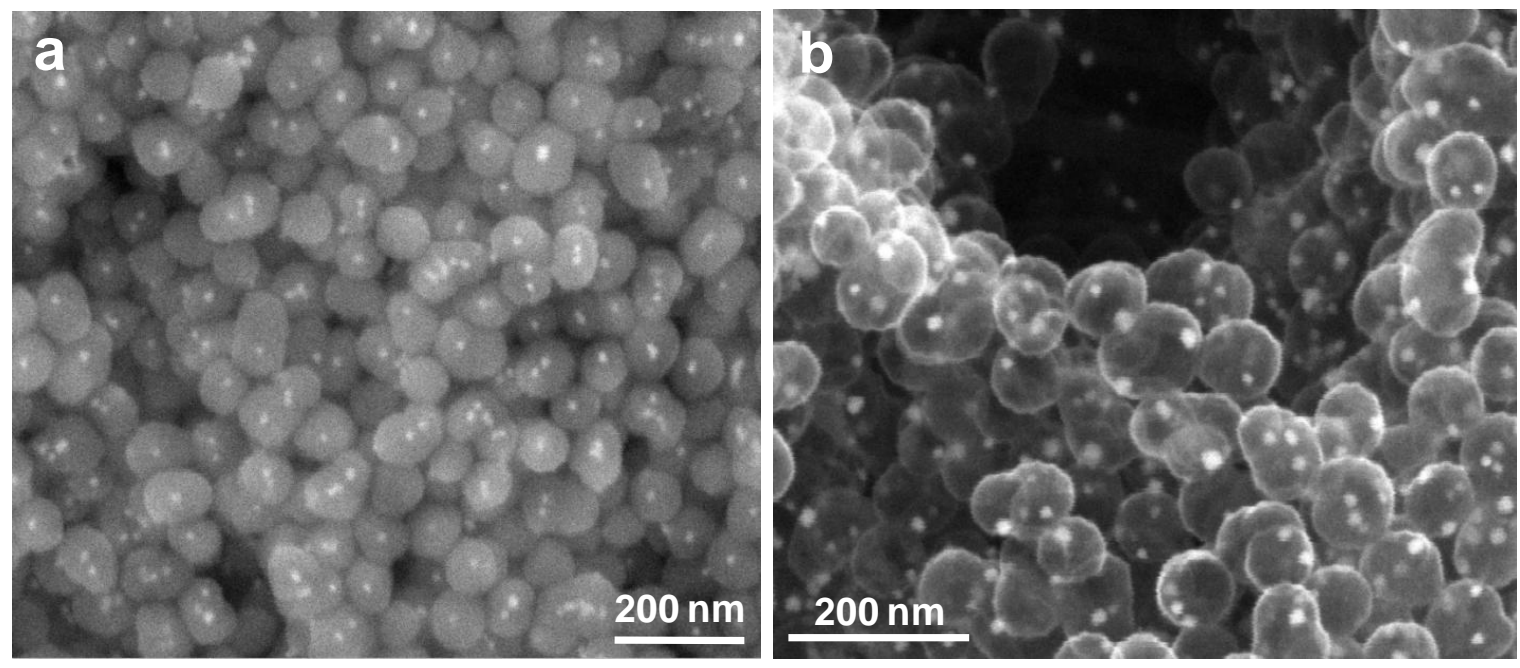

Figure 4. SEM images of yolk-structures carbon nanospheres. (a) $\mathrm{Au} @ \mathrm{SiO}_{2}$ core-shell nanospheres. (b) Au@HCS yolk-shell nanostructures.

The structural features of the Au@HCS yolk-shell nanostructures were further revealed by TEM/STEM imaging and Raman spectroscopy. Figures 5a and 5b show, respectively, the brightfield TEM and Z-contrast dark-field STEM images of a cluster of Au@HCS yolk-shell nanostructures. It is clear that the Au nanoparticles are indeed encapsulated inside the hollow spheres. High-magnification TEM imaging reveals that the Au nanoparticle is studded onto the inner surface of the hollow shell (Fig. 5c). Like the microscale HCSs, the shells of the nanoscale HCSs are graphitized and consist of around 10 graphene layers after 5 min CVD growth (Fig. 5d). However, the nanoscale HCSs contains a little higher degree of disorders than the microscale HCSs, as the stronger D Raman band shown in Fig. $3 b$. 

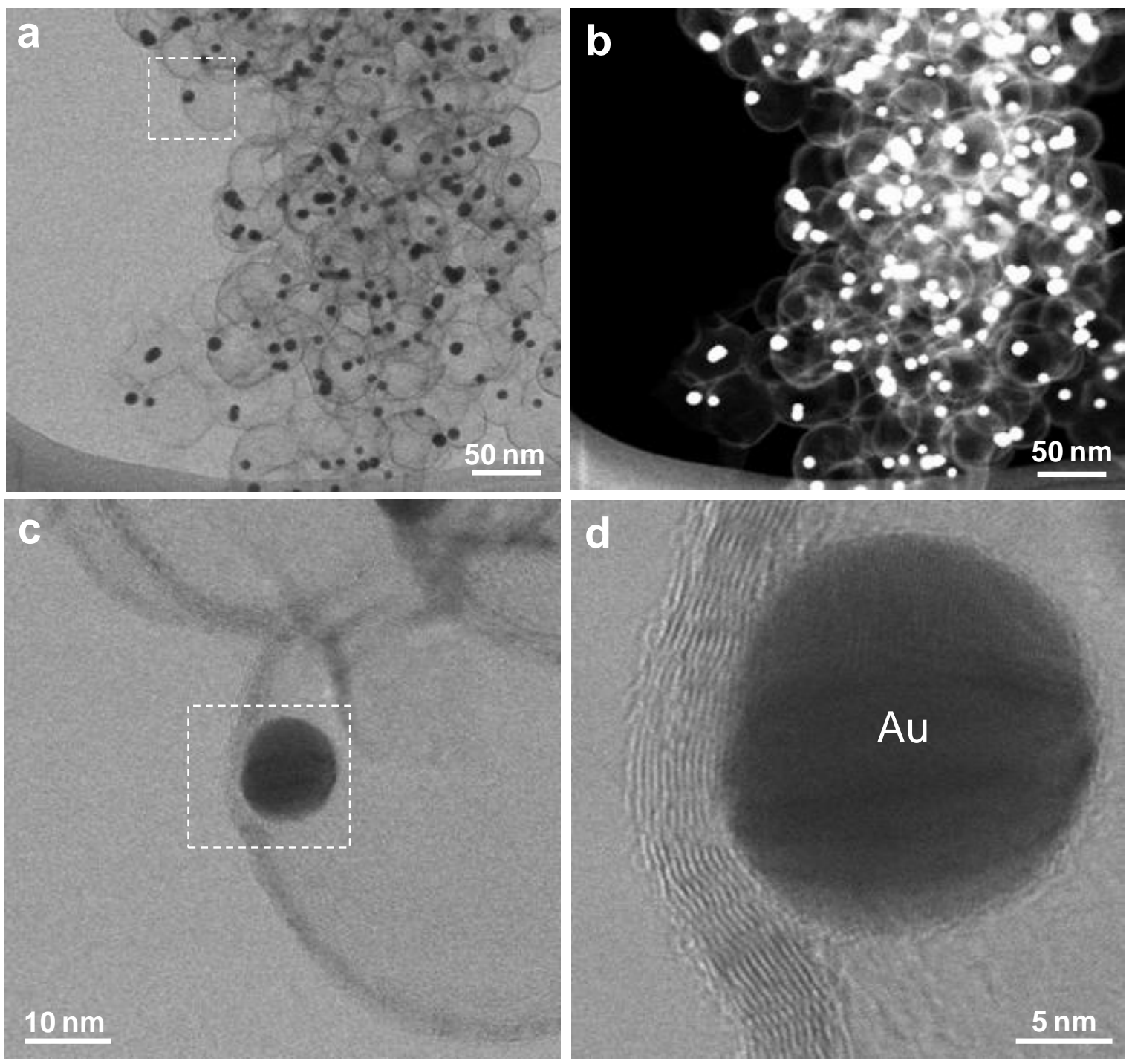

Figure 5. TEM images of yolk-structures carbon nanospheres. (a) Bright-field TEM image of Au@HCS yolk-shell nanostructures. (b) Simultaneously acquired Z-contrast dark-field STEM image of the same area in (a). (c) Magnified TEM image of the boxed area in (a). (d) HR-TEM image of the boxed area in (c). 


\section{Conclusions}

We demonstrated the synthesis of graphitized HCSs and Au@HCS yolk-shell nanostructures by metal-catalyst-free methane CVD using micro-sized $\mathrm{SiO}_{2}$ spheres and nano-sized $\mathrm{Au} @ \mathrm{SiO}_{2}$ core-shell spheres as the templates, respectively. Since the graphitization of the metal-catalystfree CVD HCSs is comparable to that of the metal-catalyzed CVD multiwall carbon nanotubes, the significance of our work also lies in the capability of growing quality graphene on silica sphere surfaces using methane CVD without the presence of metal catalysts. This finding is significant for graphene growth because, compared with the popular metal-catalyzed CVD growth [25,26], the metal-catalyst-free CVD allows the growth of graphene on technologically important dielectric substrates (including $\mathrm{SiO}_{2}$ ) for direct device fabrication, which can avoid the need for a complicated postgrowth transfer process [27]. Moreover, considering the diverse forms of $\mathrm{SiO}_{2}$ structures (e.g., spheres, plates, porous foams, etc.) and the excellent coating capability of $\mathrm{SiO}_{2}$ on other materials (e.g., metals, semiconductors, quantum dots, etc.), the metal-catalyst-free CVD technique provides a facile route to fabricate good quality, complex graphene structures with novel functionalities.

\section{Acknowledgements}

Z.W.P. thanks the support of a NSF CAREER grant (DMR-0955908). Work by S.D. and S.M.M. was supported by the U.S. Department of Energy (DOE), Office of Science, Basic Energy Sciences (BES), Chemical Sciences, Geosciences, and Biosciences Division. The TEM work was performed through a user project supported by the Oak Ridge National Laboratory's Center for Nanophase Materials Science, which is a DOE Office of Science user facility. 


\section{REFERENCES}

1. Liu J, Wickramaratne NP, Qiao SZ, Jaroniec M. Molecular-based design and emerging applications of nanoporous carbon spheres. Nat Mater 2015;14(8):763-774.

2. Zheng GY, Lee SW, Liang Z, Lee HW, Yan K, Yao HB, et al. Interconnected hollow carbon nanospheres for stable lithium metal anodes. Nat Nanotech 2014;9(8):618-23.

3. Zhang K, Zhao Q, Tao Z, Chen J. Composite of sulfur impregnated in porous hollow carbon spheres as the cathode of Li-S batteries with high performance. Nano Res 2013;6 (1):38-46.

4. You B, Yang J, Sun YQ, Su QD. Easy synthesis of hollow core, bimodal mesoporous shell carbon nanospheres and their application in supercapacitor. Chem Comm 2011;47(45):12364-6.

5. Zhang WM, Hu JS, Guo YG, Zheng SF, Zhong LS, Song WG, Wan LJ. Tin-nanoparticles encapsulated in elastic hollow carbon spheres for high-performance anode material in lithium-ion batteries. Adv Mater 2008; 20 (6):1160-5.

6. Zhao Y, Li JX, Wu CX, Ding YH, Guan LH, A yolk-shell $\mathrm{Fe}_{3} \mathrm{O}_{4} @ \mathrm{C}$ composite as an anode material for high-rate lithium batteries. ChemPlusChem 2012;77 (9):748-51.

7. Tang K, Fu LJ, White RJ, Yu LH, Titirici MM, Antonietti M, Maier J. Hollow carbon nanospheres with superior rate capability for sodium-based batteries. Adv Energy Mater 2012;2 (7):873-7.

8. Lee JS, Kim SI, Yoon JC, Jang JH. Chemical vapor deposition of mesoporous graphene nanoballs for supercapacitor. ACS Nano 2013;7(7):6047-55.

9. Wang L, Zhang J, Yang S, Sun Q, Zhu LF, Wu QM, et al. Sulfonated hollow sphere carbon as an efficient catalyst for acetalisation of glycerol. J Mater Chem A 2013;1(33):9422-6. 
10. Fang X, Liu S, Zang J, Zheng MS, Dong QF, Sun D, Zheng N. Precisely controlled resorcinol-formaldehyde resin coating for fabricating core-shell, hollow, and yolk-shell carbon nanostructures. Nanoscale 2013;5(15):6908-16.

11. Fuertes AB, Valle-Vigon P, Sevilla M. One-step synthesis of silica@ resorcinolformaldehyde spheres and their application for the fabrication of polymer and carbon capsules. Chem Commun 2012;48(49):6124-6.

12. Li N, Zhang Q, Joo JB, Lee A, Gan Y, Yin YD. Sol-gel coating of inorganic nanostructures with resorcinol-formaldehyde resin. Chem Commun 2013;49(45):5135-7.

13. Feng SS, Li W, Shi Q, Li YH, Chen JC, Ling Y, et al. Synthesis of nitrogen-doped hollow carbon nanospheres for $\mathrm{CO}_{2}$ capture. Chem Commun 2014;50(3):329-31.

14. Liu R, Mahurin SM, Li C, Unocic RR, Idrobo JC, Gao HJ, et al. Dopamine as a carbon source: the controlled synthesis of hollow carbon spheres and yolk-structured carbon nanocomposites. Angew Chem Int Ed 2011;50(3):6799-802.

15. Zhou WD, Xiao XC, Cai M, Yang L. Polydopamine-coated, nitrogen-doped, hollow carbonsulfur double-layered core-shell structure for improving lithium-sulfur batteries. Nano Lett 2014;14(9):5250-6.

16. Jayaprakash N, Shen J, Moganty SS, Corona A, Archer LA. Porous hollow carbon@ sulfur composites for high-power lithium-sulfur batteries. Angew Chem Int Ed 2011;50(26):59048.

17. White RJ, Tauer K, Antonietti M, Titirici M. Functional hollow carbon nanospheres by latex templating. J Am Chem Soc 2010;132(49):17360-3. 
18. Lu AH, Li WC, Hao GP, Spliethoff B, Bongard HJ, Schaack BB, Schuth F. Easy Synthesis of hollow polymer, carbon, and graphitized microspheres. Angew Chem Int Ed 2010;49(9):1615-8.

19. Li KY, Eres G, Howe JY, Chuang YJ, Li XF, Gu ZJ, et al. Self-assembly of graphene on carbon nanotube surfaces. Sci Rep 2013;3:2353.

20. Pan ZW, Lerch SJL, Xu L, Li XF, Chuang YJ, Howe JY, et al. Electronically transparent graphene replicas of diatoms: a new technique for the investigation of frustule morphology. Sci Rep 2014;4:6117.

21. Stöber W, Fink A, Bohn E. Controlled growth of monodisperse silica spheres in the micron size range. J Colloid Interface Sci 1968;26(1):62-9.

22. Arnal PA, Comotti M, Schüth F. High-temperature-stable catalysts by hollow sphere encapsulation. Angew Chem Int Ed 2006;45(48):8224-7.

23. Hu ZJ, Hüttinger KJ. Mechanisms of carbon deposition-a kinetic approach. Carbon 2002;40(4):624-8.

24. Pan ZW, Xie SS, Chang BH, Wang CY, Lu L, Liu W. et al. Very long carbon nanotubes. Nature 1998;394(6694):631-2.

25. Li XR, Cai WW, An JH, Kim S, Nah J, Yang DX, et al. Large-area synthesis of high-quality and uniform graphene films on copper foils. Science 2009;324(5932):1312-4.

26. Kim KS, Zhao Y, Jang H, Lee SY, Kim JM, Kim KS, et al. Large-scale pattern growth of graphene films for stretchable transparent electrodes. Nature 2009;457(7230):706-10.

27. Chen JY, Wen YG, Guo YL, Wu B, Huang LP, Xue YZ, et al. Oxygen-aided synthesis of polycrystalline graphene on silicon dioxide substrates. J Am Chem Soc 2011;133(44):1754851. 